Aspectos Éticos de la Especialidad

\title{
FORMATOS PARA \\ CONSENTIMIENTO INFORMADO
}

\author{
Alba Lucía Mondragón Cedeño* \\ Recibido: mayo 30/2002 - Revisado: julio 3/2002 - Aceptado: agosto 5/2002
}

\section{RESUMEN}

Para dar cumplimiento a la advertencia de riesgos de los tratamientos médicos y quirúrgicos ordenados en el código de Ética Médica, se han implementado diferentes modalidades a lo largo de la historia, desde anotaciones realizadas por el propio médico, hasta diferentes tipos de formatos preimpresos. En este artículo se presentan los formatos para consentimiento informado realizados y utilizados por la Asociación Vallecaucana de Obstetricia y Ginecología (Sovogin), como un aporte para ser difundidos y utilizados.

Palabras clave: consentimiento informado.

\section{SUMMARY}

Different models for informed consent are used in the country. The models for informed consent of the Asociación Vallecaucana de Obstetricia y Ginecología (Sovogin), are presented here with the purpose of promote them and increasing their use.

Key words: informed consent.

\footnotetext{
* Ginecóloga, Universidad del Valle; Medicina de laReproducción, Ginebra, Suiza; Asociación Vallecaucana de Obstetricia y Ginecología. Correspondencia: Alba Lucía Mondragón C. Calle 25 No. 2 n - 57, consultorio 506. Clínica de Los Remedios, Cali; tel. 6815476 - 6081000 ext. 556. Correo electrónico: albamon@hotmail.com
}

\section{INTRODUCCIÓN}

El Código de Ética Médica contempla la importancia de advertir sobre los riesgos de los tratamientos médicos y quirúrgicos a realizar. A través del tiempo esto se ha hecho en diferentes formas y todavía en algunas instituciones no se realiza. Inicialmente sólo se hacia en forma verbal, luego en forma escrita dentro de la historia clínica por el médico tratante y, ocasionalmente, por otro profesional de la salud (psicóloga, enfermera o trabajadora social). Luego aparecieron los formatos preimpresos; inicialmente estos formatos eran para consentimientos generales firmados por el paciente al ingreso a una institución, en los cuales no se consignaban los riesgos específicos de los procedimientos, por lo cual no tenían ninguna validez legal. Recientemente, la Sociedad Colombiana de Obstetricia y Ginecología SCOG, difundió unos formatos elaborados por el doctor Guido Parra y colaboradores, donde se hace advertencia de riesgos específicos.

En este articulo se presentan, previa autorización de la junta directiva de Sovogin y su actual presidente, doctor Edgar Iván Ortiz L., los modelos de formatos de consentimiento informado que fueron elaborados hace cuatro años por la Asociación Vallecaucana de Obstetricia y Ginecología, y que fueron difundidos para su uso hace dos años y 
reformados de acuerdo a sugerencias de los usuarios después de su aplicación, como un aporte para ser difundidos y utilizados. La unificación de los modelos será un proyecto futuro.

\section{RESEÑA HISTÓRICA}

En 1997 la Junta Directiva de Sovogin, integrada en ese momento por los doctores María Cecilia Arturo R., Farid Amastha H., Cesar Prieto A., Hoover Canaval E., Fernando Zuluaga A., Alba Lucía Mondragón C., Carlos Díaz R., Jaime Messa A. y Gustavo Vásquez Z., inició un proyecto para realizar formatos de consentimiento informado específicos para las diferentes entidades de uso diario en ginecología y obstetricia. Se invitó a participar a las diferentes subespecialidades de la región y diferentes ginecólogos, conformando varios grupos de trabajo. Se contó con la asesoría permanente de la doctora Bertha Lucy Cevallos, abogada con amplia experiencia en el campo médico, en ese momento integrante del grupo jurídico de Fepasde, regional Valle. Los borradores de los formatos fueron discutidos en mesas de trabajo de acuerdo con los temas, algunas de ellas en otras ciudades del departamento, con participación de especialistas de esas ciudades, algunos de ellos con título de abogado, para finalmente producir los formatos iniciales que fueron publicados y difundidos hace dos años.

Los formatos han tenido modificaciones derivadas de sugerencia de los usuarios, dando como producto final los formatos que actualmente reposan en nuestra asociación y que son usados en los consultorios privados y algunas instituciones de salud como Profamilia y el Seguro Social de Cali.

\section{JUSTIFICACIÓN}

La legislación colombiana contempla la constancia que por Ley debe contener la historia clínica. En la Ley 23 de 1981 o Código de Ética Médica, se consagran las actividades y advertencias que el médico debe cumplir, en los siguientes términos:
Articulo 15: El médico no expondrá a sus pacientes a riesgos injustificados. Pedirá su consentimiento para aplicar los tratamientos médicos y quirúrgicos que considere indispensables y que puedan afectarlo física o psíquicamente, salvo en los casos en que ello no fuere posible, y le explicará al paciente o a sus responsables de tales consecuencias anticipadamente.

Articulo 16: la responsabilidad del médico por reacciones adversas, inmediatas o tardías, producidas por efecto del tratamiento, no irá más allá del riesgo previsto. El médico advertirá de él al paciente $\mathrm{o}$ a sus familiares $\mathrm{o}$ allegados.

Como complemento de estas normas, el Decreto 3380 de 1981, dispone:

Articulo 10: el médico cumple la advertencia del riesgo previsto, a que se refiere el inciso 2 del articulo 16 de la Ley 23 de 1981, con el aviso que en forma prudente haga a sus familiares y allegados, con respecto a los efectos adversos que, en su concepto, dentro del campo de la práctica médica, pueden llegar a producirse como consecuencia del tratamiento o procedimiento médico.

Artículo 11: el médico quedará exonerado de hacer la advertencia del riesgo previsto en los siguientes casos:

a) Cuando el estado mental del paciente y la ausencia de parientes o allegados se lo impidan.

b) Cuando exista urgencia o emergencia para llevar a cabo el tratamiento o procedimiento médico.

Artículo 12: el médico deja constancia en la historia clínica del hecho de la advertencia del riesgo previsto o de la imposibilidad de hacerla.

En nuestra experiencia de varios años, en muy pocos casos se ha alterado la adhesión a un tratamiento al conocer esos riesgos.

Aunque la ley no es explicita en que se debe dejar constancia del rechazo de un tratamiento, también se diseño un formato para dejar constancia de 
la no-aceptación de algunas conductas médicas, como la remisión a otra institución o la autopsia.

\section{SUGERENCIAS DE UTILIZACIÓN}

El formato anexo debe ser entregado por el médico con tiempo suficiente para ser leído y comprendido por la usuaria potencial y debe acompañarse de explicaciones verbales en lenguaje sencillo de cómo la ley ordena que se otorgue consentimiento previo al procedimiento propuesto.

También se recomienda acompañar el formato con múltiples explicaciones educativas del procedimiento y del problema que presenta la paciente, en términos accesibles al lenguaje común de las usuarias, aclarando los beneficios y riesgos, ya sea en forma verbal o por medio de cartillas, videos y/ o conferencias.
Se debe tener un tiempo adicional para resolver las preguntas que surjan a fin de que no queden dudas. Se debe informar, también, que en el futuro se puede cambiar de opinión y revocar el consentimiento por simple decisión.

El formato debe ser firmado y anexado a la historia clínica, consignando en ella la fecha de la anexión.

\section{CONCLUSIONES}

Es necesario dar cumplimiento a la ley referente a la advertencia de riesgos. A continuación se ponen a disposición los formatos de consentimiento informado elaborados y utilizados por la Asociación Vallecaucana de Obstetricia y Ginecología, para su amplia difusión y utilización. La unificación de los formatos a nivel nacional se sugiere sea un proyecto futuro. 


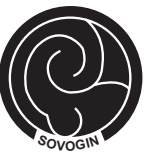

\section{CONSULTA GINECOLÓGICA}

(En cumplimiento del programa educativo de Sovogin)

(Nombre completo)

En este acto médico, el(la) doctor(a) previo análisis de mis antecedentes, datos de la historia clínica y del examen físico, me ordenará exámenes diagnósticos para aclarar el problema por el cual consulté. Posteriormente me hablará del(los) tratamiento(s) o procedimiento(s) que considere idóneos para tratar de solucionarlo o aliviarlo y de los riesgos previsibles de éstos.

Entiendo que si no informo adecuadamente y con la verdad todos los datos necesarios, se pueden ocasionar confusiones en el diagnóstico o errores en la selección de los tratamientos, sin que estos resultados sean atribuibles al médico. Así como si decido no realizar los exámenes diagnósticos ordenados, o no cumplo los tratamientos ordenados y/o no acepto las intervenciones sugeridas por mi médico tratante, pueden presentarse reacciones adversas, ajenas al actuar de mi médico, sin que ello signifique pérdida de mis derechos a la atención profesional posterior.

Firma

C.C. de

Ciudad y fecha 


\title{
CONSENTIMIENTO INFORMADO PARA DONACIÓN DE SEMEN PROGRAMAS DE REPRODUCCIÓN ASISTIDA FORMATO PARA EL DONANTE
}

\author{
(En cumplimiento de la Ley 23 de 1981)
}

\section{(Nombre)}

en forma voluntaria y sin ninguna presión o inducción consiento en donar una o varias muestras de semen y consiento en que el espécimen de semen que estoy donando sea usado con el propósito de producir embarazo por medio de inseminación artificial en las pacientes seleccionadas por

Afirmo que no intentaré encontrar la identidad de las personas para las cuales yo estoy haciendo la donación y entiendo que mi propia identidad será guardada en secreto y en estricta confidencia, a menos que alguna corte ordene abrirla por una buena causa mostrada y puntualizada por la ley.

Entiendo el método para coleccionar la muestra, la recogeré siguiendo estrictamente las instrucciones antes de cada donación y la proporcionaré el día y la hora acordada. Entiendo que seré compensado económicamente solamente por un espécimen aceptable y que no falte a las condiciones especificadas para calidad y cantidad.

Una vez que esté participando como donante en un caso de inseminación artificial reportaré todo cambio significante en mi salud, especialmente en lo referente a enfermedades venéreas, y reportaré cualquier información nueva acerca de factores genéticos de los cuales no hubiera tenido conocimiento durante el proceso de tamizaje, aunque éstos no constituyan un cambio en mi estado de salud. Consiento en ser contactado periódicamente aun después de la terminación de un ciclo de inseminación artificial, con el fin de proveer información referente a los parámetros anteriores. Me comprometo a tener informado al programa de los cambios de domicilio durante un largo periodo.

Entiendo que puedo terminar mi participación como donante en cualquier momento con la apropiada notificación a la oficina del programa y acepto que puedo ser excluido como donante del programa en cualquier momento.

En tales condiciones consiento donar una o varias muestras de semen para ser utilizadas en procedimientos de REPRODUCCIÓN ASISTIDA.

Firma

C.C.

Ciudad y fecha

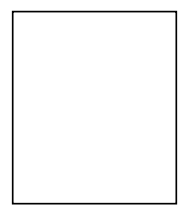

Índice derecho 


\section{CONSENTIMIENTO INFORMADO PARA CERCLAJE}

(En cumplimiento de la Ley 23 de 1981)

(Nombre)

en forma voluntaria consiento en que el (la) doctor (a)

y el ayudante que el (ella) designe me realicen CERCLAJE para tratar de prevenir un aborto.

Entiendo que este procedimiento consiste básicamente en la colocación de unos puntos de sutura alrededor del cuello uterino, tratando de cerrarlo para que el bebé permanezca adentro y evitar que se produzca un aborto.

Esta cirugía no garantiza el nacimiento de un bebé de término o completamente sano, se me ha explicado que la garantía no es total pues la práctica de la medicina y cirugía no son una ciencia exacta. Entiendo que todo lo que harán será colocar los puntos muy cuidadosamente haciendo lo mejor tanto para la madre como para el feto, debiendo mi médico colocar todo su conocimiento y su pericia en buscar obtener el mejor resultado.

He sido informada y entiendo que como en toda cirugía y por causas independientes del actuar de mi médico se pueden presentar complicaciones comunes y potencialmente serias que podrían requerir tratamientos complementarios tanto médicos como quirúrgicos, siendo las complicaciones de un cerclaje: contracciones uterinas, sangrado, infecciones, desgarros del cuello uterino, ruptura de la bolsa amniótica con pérdida fetal y parto prematuro. También se me informa la posibilidad de complicaciones severas como: pelvi peritonitis, ruptura uterina, hemorragia, trombosis o muerte que aunque son poco frecuentes representan como en toda intervención quirúrgica un riesgo excepcional de perder la vida derivado del acto quirúrgico o de la situación vital de cada paciente.

En mi caso particular, el (la) doctor (a) me ha explicado que presento los siguientes riesgos adicionales:

Entiendo que para este procedimiento se necesita anestesia, la cual se evaluará y realizará por el servicio de anestesia.

Yo he entendido sobre las condiciones y objetivos de la cirugía que se me va a practicar, los cuidados que debo tener antes y después, estoy satisfecha con la información recibida del médico tratante quien me ha dado la oportunidad de preguntar y resolver las dudas y todas ellas han sido resueltas a satisfacción, además comprendo y acepto el alcance y los riesgos justificados de posible previsión que conlleva este procedimiento quirúrgico que aquí autorizo. En tales condiciones consiento que se me realice CERCLAJE.

Firma del paciente

C.C.

Ciudad y fecha

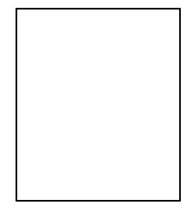

Índice derecho 


\section{CONSENTIMIENTO INFORMADO PARA OPERACIÓN CESÁREA}

(En cumplimiento de la Ley 23 de 1981)

(Nombre)

en forma voluntaria, consiento en que el (la) doctor (a)

como cirujano (a), y el ayudante que él (ella) designe, me realicen operación CESÁREA, por indicación médica.

Entiendo que ese procedimiento consiste básicamente en la extracción de mi hijo por una apertura quirúrgica en la pared anterior de mi abdomen y que su justificación es debida a que en las circunstancias actuales de mi embarazo consistentes en

hacen presumir un mayor riesgo, para mi y/o para mi bebé, durante un parto vaginal.

Se me ha explicado que como en todo acto médico no puede existir garantía en los resultados, por no ser la medicina una ciencia exacta, debiendo mi médico colocar todo su conocimiento y su pericia para buscar obtener el mejor resultado.

Entiendo que como en toda intervención quirúrgica y por causas independientes del actuar de mi médico, se pueden presentar complicaciones comunes y potencialmente serias que podrían requerir tratamientos complementarios, tanto médicos como quirúrgicos, siendo las complicaciones más frecuentes de la cesárea: infecciones (urinarias, uterinas, pélvicas, abdominales, de la herida, etc.), hipotonía uterina (no contracción del útero) y hemorragias con la posible necesidad de transfusión sanguínea intra o posoperatoria, seromas (acumulación de líquido en la herida), hematomas (moretones). Existen otras complicaciones de menor frecuencia que requieren otras cirugías inmediatas (histerectomía o laparotomía), o posteriores tales como: eventraciones (hernias), adherencias, dehiscencia de las suturas y otras. También se me informa la posibilidad de complicaciones severas como pelvi peritonitis, ruptura uterina en los siguientes embarazos, trombosis o muerte que, aunque son poco frecuentes representan, como en toda intervención quirúrgica, un riesgo excepcional de morir derivado del acto quirúrgico o de la situación vital de cada paciente.

En mi caso particular, el (la) doctor (a) me ha explicado que presento los siguientes riesgos adicionales:

Entiendo que para esta cirugía se requiere de anestesia, cuya realización está a cargo del servicio de anestesia y sus riesgos serán valorados y considerados por el médico (a) anestesiólogo (a). Se me informa la necesidad de la presencia de un médico capacitado para la atención de mi recién nacido, quien ejercerá un acto médico independiente al cirujano obstetra.

Manifiesto que estoy satisfecha con la información recibida del médico tratante, quien me ha dado la oportunidad de preguntar y resolver las dudas y todas ellas han sido resueltas a satisfacción. Yo he entendido sobre las condiciones y objetivos de la cirugía que se me va a practicar, los cuidados que debo tener antes y después de ella, además comprendo y acepto el alcance y los riesgos justificados de posible previsión que conlleva el procedimiento quirúrgico que aquí autorizo. En tales condiciones consiento en que se me realice OPERACIÓN CESÁREA.

Firma del paciente

C.C.

Ciudad y fecha

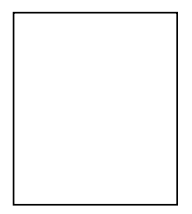

Índice derecho 


\section{CONSENTIMIENTO INFORMADO PARA CIRUGÍA LAPAROSCÓPICA}

(En cumplimiento de la Ley 23 de 1981)

(Nombre)

en forma voluntaria, consiento en que el (la) doctor (a) :

como cirujano(a) y el (la) ayudante que él (ella) designe, me realicen cirugía laparoscópica, como tratamientopara:

Entiendo que esta cirugía consiste básicamente en la introducción de gas a través del abdomen y luego la colocación de un tubo por el ombligo que contiene un instrumento óptico para ver en el interior y por medio de otros orificios en diferentes sitios, la colocación de pinzas e instrumentos quirúrgicos para realizar la cirugía observando la imagen proyectada en un televisor.

Se me ha explicado y entiendo que como en todo acto médico no puede existir garantía en los resultados, pues la práctica de la medicina y cirugía no son una ciencia exacta, debiendo mi médico colocar todo su conocimiento y pericia en obtener el mejor resultado.

También he entendido que para mi problema, existen otros tipos de tratamiento tales como:

y voluntariamente he elegido la cirugía laparoscópica.

Yo autorizo para que mi cirugía sea observada con fines didácticos, ya sea directamente o en el vídeo que se grabará de mi cirugía, el cual no será identificado con mi nombre, pero sí usado por fuera de mi historia clínica médica.

Entiendo que para esta cirugía se necesita anestesia, la cual se evaluará y realizará por el servicio de anestesia.

Entiendo que como en toda intervención quirúrgica y por causas independientes del actuar de mi médico se pueden presentar complicaciones comunes y potencialmente serias que podrían requerir tratamientos complementarios, tanto médicos como quirúrgicos, siendo las complicaciones más frecuentes: náuseas, vómito, dolor o problemas urinarios, sangrado, infección, reacciones alérgicas o retención urinaria, heridas involuntarias en el útero, tubas, ovarios, intestino, vasos sanguíneos, vejiga u otros órganos. También se me informa la posibilidad de complicaciones severas como histerectomía (perdida del útero), colostomía (comunicación del intestino a la piel del abdomen), hemorragia severa, parálisis o muerte que aunque son poco frecuentes representan, como en toda intervención quirúrgica, un riesgo excepcional de perder la vida, derivado del acto quirúrgico o de la situación vital de cada paciente.

En mi caso particular, él (la) médico (a) me ha explicado que presento los siguientes riesgos adicionales:

Yo entiendo los cuidados que debo tener antes y después de esta cirugía, estoy satisfecha con la información recibida del médico tratante, quien lo ha hecho en un lenguaje claro y sencillo y me ha dado la oportunidad de preguntar y resolver las dudas y todas ellas han sido resueltas a satisfacción, además comprendo y acepto el alcance y los riesgos justificados de posible previsión que conlleva este procedimiento quirúrgico que aquí autorizo. En tales condiciones consiento que se me realice CIRUGÍA LAPAROSCÓPICA.

Firma del paciente

C.C.

Ciudad y fecha

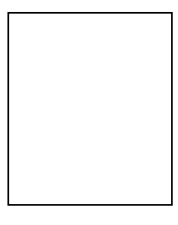

Índice derecho 


\section{CONSENTIMIENTO INFORMADO PARA CIRUGÍA DE CÉRVIX}

(En cumplimiento de la Ley 23 de 1981)

(Nombre)

en forma voluntaria consiento en que el (la) doctor (a)

me realice cirugía de cérvix por presentar cambios en la citología que necesiten aclarar y/o por presentar cambios en la biopsia de cérvix que necesiten tratamiento.

En mi caso particular el médico me ha dicho que va a realizarme:

Entiendo que ese procedimiento consiste básicamente en la exploración del cérvix con un aparato de aumento llamado colposcopio. Según los hallazgos se hará extirpación de las estructuras que estén afectadas por medio de la toma de biopsia o resección de una porción redondeada del cérvix en forma de cono (conización). Dependiendo del criterio médico, también se pueden hacer cauterizaciones que consisten en una destrucción de la superficie del cérvix ya sea con sustancias químicas o con frío (criocauterio).

Esta cirugía no garantiza la total identificación y/o desaparición de mi problema, ni evita que en el futuro sean necesarias nuevas intervenciones para solucionar problemas residuales o complicaciones. Se me ha explicado que la garantía no es total pues la práctica de la medicina y cirugía no son una ciencia exacta, debiendo mi médico colocar todo su conocimiento y su pericia en buscar obtener el mejor resultado.

Entiendo que como en toda intervención quirúrgica se pueden presentar complicaciones comunes y potencialmente serias que podrían requerir tratamientos complementarios tanto médicos como quirúrgicos tales como: sangrado o hemorragias, con la posible necesidad de transfusión (intra o postoperatoria) que requieran para su control taponamientos vaginales por algún tiempo y/o cirugías para contener la hemorragia, infecciones con posible evolución febril, reacciones alérgicas, mala cicatrización (estenosis o estrechez o al contrario dilatación permanente, lo que puede producir problemas para un futuro embarazo), perforaciones involuntarias de órganos vecinos como vejiga, intestino, colon, vasos sanguíneos, fístulas urinarias o intestinales, persistencia del problema, reaparición del problema en el mismo o sitio o en otras partes. La posibilidad de complicaciones severas como histerectomía, trombosis o muerte son raras, pero como en toda intervención quirúrgica existe un riesgo excepcional de morir derivado del acto quirúrgico o de la situación vital de cada paciente.

Entiendo que el material que se saque se someterá a estudio anatomopatológico posterior en el laboratorio de patología de , siendo mi deber el reclamar su resultado e informarlo al médico.

Yo he entendido los cuidados que debo tener antes y después, estoy satisfecha con la información recibida del médico tratante, quien me ha dado la oportunidad de preguntar y resolver las dudas y todas ellas han sido resueltas a satisfacción, además comprendo y acepto el alcance y los riesgos justificados de posible previsión que conlleva este procedimiento quirúrgico que aquí autorizo. En tales condiciones consiento que se me realice CIRUGÍA DE CÉRVIX.

Firma del paciente

C.C. expedida en

Ciudad y fecha 


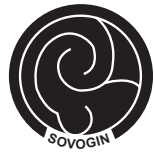

\section{CONSULTA Y ESTUDIO DE INFERTILIDAD}

(En cumplimiento del programa educativo de Sovogin)

(Nombre femenino)

y (Nombre masculino)

encalidad de:

En este acto médico el(la) doctor(a)

previo análisis de sus antecedentes, datos de la historia clínica y del examen físico, les ordenará exámenes diagnósticos para tratar de encontrar la causa por la cual posiblemente no logran un embarazo.

Si no informan adecuadamente y con la verdad todos los datos necesarios, se pueden ocasionar confusiones en él diagnóstico o errores en la selección de los tratamientos sin que estos resultados sean atribuibles a su médico.

Algunos resultados pueden ser demorados, algunos incómodos o le pueden producir dolor. En ocasiones no se encuentra la causa de infertilidad con la inmediatez esperada y serán necesarios otros exámenes más complejos. En un porcentaje importante no se puede encontrar la causa, declarándose una infertilidad inexplicable. Algunas pacientes se pueden embarazar durante el transcurso de la realización de dichos exámenes.

Entendemos todo lo arriba escrito y también entendemos que si decidimos no realizar los exámenes diagnósticos y/o los tratamientos ordenados, las consecuencias son ajenas del actuar de mi médico sin que ello signifique pérdida de mis derechos a la atención profesional posterior.

Firmas

C.C. C.C.

Ciudad y fecha 


\section{CONTROL PRENATAL}

(En cumplimiento del programa educativo de Sovogin)

(Nombre completo)

En este acto médico el (la) doctor(a)

me realizará la atención y el control prenatal de mi actual proceso de gestación.

Entiendo que si no informo adecuadamente y con la verdad todos los datos necesarios, se puede ocasionar confusión en el diagnóstico o error en la selección del tratamiento que busca mi bienestar y el de mi hijo, sin que estos resultados sean atribuibles a mi médico.

En todo proceso de gestación pueden ocurrir complicaciones previsibles, entre otras: aborto espontáneo (en un 20\%), defectos físicos o mentales (4\%), parto pretérmino (bebé nacido antes de los 9 meses), toxemia (edema progresivo con aumento de la tensión arterial y daño progresivo de órganos), placenta previa, desprendimiento prematuro de la placenta, accidentes del cordón umbilical, malposición fetal, embolismo (porciones de líquido o sangre en el pulmón), ruptura uterina, toxemia complicada (hemorragias, convulsiones, problemas de coagulación y muerte) y otras que son raras en el embarazo pero existiendo un riesgo de muerte materna y/o fetal derivado del proceso de gestación o de la situación vital de cada paciente.

En mi caso particular mi médico tratante me ha explicado que presento los siguientes riesgos adicionales:

Todos estos riesgos y complicaciones son independientes de la calidad del control médico.

Yo entiendo este acto médico y acepto los riesgos arriba explicados y entiendo que si no acepto las intervenciones sugeridas por mi médico tratante y/o no cumplo las citas de control y/o decido no realizar los exámenes diagnósticos y/o los tratamientos ordenados, pueden presentarse reacciones adversas, ajenas al actuar de mi médico, sin que ello signifique pérdida de mis derechos a la atención profesional posterior.

Firmas: CC

C.C. En calidad de

C.C.

Ciudad y fecha 


\section{CONSENTIMIENTO INFORMADO PARA HISTERECTOMÍA}

(En cumplimiento de la Ley 23 de 1981)

(Nombre)

consiento en que el (la) doctor (a)

como cirujano(a) y el (la) ayudante que él (ella) designe, me realicen HISTERECTOMÍA como tratamiento para

Entiendo que esta cirugía consiste básicamente en la extirpación del útero con o sin el cuello, lo que supone la imposibilidad de tener hijos, así como la ausencia de menstruaciones. La histerectomía puede llevar asociadas la extirpación de los anexos (ovarios y trompas) según edad, patología asociada y criterio médico en el momento de la intervención. Al extirpar los ovarios se instaura la menopausia, pudiendo recibir terapia hormonal sustitutiva posteriormente, según indicación médica. Esta cirugía puede realizarse por: vía laparoscópica, vía vaginal, o comúnmente vía abdominal (apertura quirúrgica en la cara anterior del abdomen). Entiendo que si se inicia la cirugía vía vaginal, ante los hallazgos o circunstancias de la cirugía, existe la posibilidad de que el cirujano se vea en la necesidad de proceder a continuar vía abdominal dependiendo del criterio médico.

Se me ha explicado y entiendo que no es posible garantizar resultado alguno pues la práctica de la medicina y cirugía no son una ciencia exacta, debiendo mi médico colocar todo su conocimiento y su pericia para buscar obtener el mejor resultado.

También he entendido que existen otros tipos de tratamientos, por ejemplo: miomectomía para los miomas (sacar solo el tumor), tratamiento hormonal para hemorragias o endometriosis (dar medicamentos tomados o inyectados) y en mi caso particular

los cuales no acepto y voluntariamente he elegido HISTERECTOMÍA.

Entiendo claramente que esta operación me dejará con una permanente incapacidad para tener hijos, y acepto la infertilidad producida por ella y en caso de que sea necesario extirparme los ovarios acepto las consecuencias de la ausencia de las hormonas que ellos producen.

Entiendo que para esta cirugía se necesita anestesia, la cual se evaluará y realizará por el servicio de anestesia.

Entiendo que la pieza extirpada se someterá a estudio anatomopatológico posterior

en siendo mi deber el reclamar su resultado e informarlo al médico.

Entiendo que como en toda intervención quirúrgica, y por causas independientes del actuar de mi médico, se pueden presentar complicaciones comunes y potencialmente serias, que podrían requerir de tratamientos complementarios médicos o quirúrgicos, siendo las complicaciones más frecuentes de la histerectomía: náuseas, vómito, dolor, inflamación, moretones, seromas (acumulación de líquido en la cicatriz), granulomas en piel o en vagina (reacción a cuerpo extraño o sutura), queloide (crecimiento excesivo de la cicatriz), hematomas, sobre todo en la cúpula vaginal (acumulación de sangre), cistitis, retención urinaria, sangrado o hemorragias con la posible necesidad de transfusión (intra o posoperatoria), infecciones con posible evolu- 
ción febril (abscesos de cúpula, urinarios, de pared abdominal, pélvicas, reacciones alérgicas, íleo paralítico (acumulación de gases y líquido en el intestino) y anemia, heridas o quemadura por bisturí eléctrico involuntarias en vasos sanguíneos, vejiga u otros órganos o para solucionar problemas tardíos como dolor pélvico, adherencias, fístulas (escape involuntario de orina o materia fecal por la vagina), eventración (hernias en la cicatriz), prolapsos (descensos) de cúpula vaginal, quistes en los ovarios (cuando se conservan los anexos) y obstrucciones en el uréter (conducto que lleva la orina hasta la vejiga). La posibilidad de complicaciones severas como pelviperitonitis (infección generalizada en el abdomen), ligadura de uréter con pérdida renal, heridas u obstrucción de arteria ilíaca con compromiso de la circulación de la pierna (amputación), trombosis o muerte son raras, pero como en toda intervención quirúrgica, representan un riesgo excepcional de perder la vida derivado del acto quirúrgico o de la situación vital de cada paciente.

En mi caso particular, el (la) doctor (a) me ha explicado que presento los siguientes riesgos adicionales:

Yo he entendido los cuidados que debo tener antes y después de la cirugía, estoy satisfecha con la información recibida del médico tratante, quien lo ha hecho en un lenguaje claro y sencillo, me ha dado la oportunidad de preguntar y resolver las dudas, y todas ellas han sido resueltas a satisfacción. Además comprendo y acepto el alcance y los riesgos justificados de posible previsión que conlleva este procedimiento quirúrgico que aquí autorizo. En tales condiciones consiento que se me realice HISTERECTOMÍA.

Firma del paciente

C.C.

Ciudad y fecha

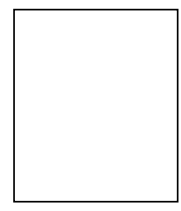

Índice derecho 


\section{CONSENTIMIENTO INFORMADO PARA CIRUGÍA DE INCONTINENCIA URINARIA}

(En cumplimiento de la Ley 23 de 1981)

(Nombre)

en forma voluntaria consiento en que el (la) doctor (a)

y el ayudante asignado, me realicen cirugía PARA INCONTINENCIA URINARIA, por presentar escape involuntario de la orina por la uretra.

Entiendo que ese procedimiento consiste básicamente en la elevación de la vejiga por medio de unos puntos de sutura los cuales pueden realizase por vía vaginal, vía abdominal, vía laparoscópica, vía transuretral o en forma combinada abdominal y vaginal, dependiendo del criterio médico y de los recursos técnicos que tenga la institución.

Esta cirugía no garantiza la total desaparición de mi problema, ni evita que en el futuro sean necesarias nuevas intervenciones para solucionar problemas residuales o recidivas. Se me ha explicado que la garantía no es total pues la práctica de la medicina y cirugía no son una ciencia exacta, debiendo mi médico colocar todo su conocimiento y su pericia en buscar obtener el mejor resultado.

Entiendo que como en toda intervención quirúrgica se pueden presentar complicaciones comunes y potencialmente serias que podrían requerir tratamientos complementarios tanto médicos como quirúrgicos tales como: náuseas, vómito dolor, inflamación, moretones, seromas (acumulación de líquido en la cicatriz), granulomas (reacción a cuerpo extraño o sutura), queloide (crecimiento excesivo de la cicatriz), hematomas (acumulación de sangre), sangrado o hemorragias con la posible necesidad de transfusión (intra o posoperatoria), infecciones con posible evolución febril (urinarias, de piel, abscesos, peritonitis), reacciones alérgicas, anemia, perforaciones involuntarias de órganos como vejiga, uretra, intestino, colon, vasos sanguíneos, fístulas urinarias o intestinales, hernias abdominales, adherencias, obstrucción intestinal por bridas, obstrucción de la uretra y vagina con necesidad de permanecer con sonda vesical por tiempo prolongado y dificultades para las relaciones sexuales, persistencia de la incontinencia o reaparición en el futuro inmediato o tardío. La posibilidad de complicaciones severas como septicemia (infección generalizada), ligadura de grandes vasos, uréter, uretra, trombosis o muerte son raras, pero como en toda intervención quirúrgica, existe un riesgo excepcional de morir derivado del acto quirúrgico o de la situación vital de cada paciente.

En mi caso particular, el (la) doctor (a) me ha explicado que presento los siguientes riesgos adicionales:

Entiendo que para esta cirugía se necesita anestesia, la cual se evaluará y realizará por el servicio de anestesia.

Yo he entendido los cuidados que debo tener antes y después, estoy satisfecha con la información recibida del médico tratante, quien me ha dado la oportunidad de preguntar y resolver las dudas, y todas ellas han sido resueltas a satisfacción; además, comprendo y acepto el alcance y los riesgos justificados de posible previsión que conlleva el procedimiento quirúrgico que aquí autorizo. En tales condiciones consiento que se me realice CIRUGÍA PARA INCONTINENCIA URINARIA.

Firma del paciente

C.C. expedida

Ciudad y fecha

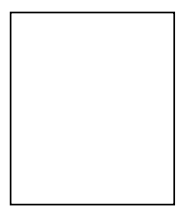




\section{CONSENTIMIENTO INFORMADO PARA INDUCCIÓN DEL PARTO}

(En cumplimiento de la Ley 23 de 1981)

(Nombre completo de la paciente)

En forma voluntaria y sin ninguna presión, consiento en que el (la) doctor (a)

y el personal paramédico asignado, me realicen y controlen la INDUCCIÓN MÉDICA DEL PARTO.

Entiendo que ese procedimiento consiste básicamente en provocar la finalización del embarazo, por causas medicas u obstétricas, lo cual se puede realizar de diferentes formas, dependiendo de las características de cada caso, quedando a juicio del médico tanto su indicación como la elección del método empleado.

La formas más frecuentes de realizar esta inducción son:

1. Rotura de la bolsa amniótica.

2. Administración intracervical de gel de prostaglandinas o tabletas, que se utiliza para mejorar la maduración del cuello uterino.

3. Administración intravenosa de oxitocina mediante goteo, que puede realizarse directamente o después de alguno de las anteriores.

Este procedimiento terminará con el nacimiento de un nuevo bebé. ESTA ATENCIÓN MÉDICA NO GARANTIZA EL NACIMIENTO DE UN BEBÉ COMPLETAMENTE SANO; se me ha explicado que la garantía no es total pues la práctica de la medicina y la cirugía no son ciencias exactas y que por lo tanto no se puede garantizar resultados que no dependen exclusivamente del médico. El personal asignado debe colocar todo su conocimiento y su pericia para buscar obtener el mejor resultado.

He sido informada y entiendo que la inducción del parto es ampliamente utilizada y sus riesgos no son elevados, pero que como en cualquier procedimiento médico puede condicionar a complicaciones en la madre o en el feto, siendo las más importantes:

1. Infección materna o fetal, cuyo riesgo aumenta principalmente a partir de las 24 horas de la rotura de la bolsa amniótica.

2. Aparición de sufrimiento fetal agudo, por disminución del aporte de oxígeno del feto durante las contracciones uterinas.

3. Fracaso de la inducción.

4. Ruptura uterina, complicación cuyo riesgo aumenta cuando se administra oxitocina o prostaglandinas y que supone un grave riesgo para la vida de la madre y del feto.

5. Prolapso de cordón, complicación que puede ocurrir tras la rotura de la bolsa amniótica y que pone en grave peligro la vida fetal.

6. Falta de contracción uterina después del parto o cesárea con hemorragia severa.

La aparición de alguna de estas complicaciones conlleva habitualmente a la realización de una cesárea abdominal para salvaguardar la vida de la madre o del niño y, en caso de ruptura uterina o falta de contracción 
uterina con hemorragia, será necesario la extracción de la matriz (histerectomía) quedando con una imposibilidad permanente de tener hijos (esterilidad).

La posibilidad de complicaciones severas como histerectomía, pelvi-peritonitis, ruptura uterina, trombosis o muerte son raras, pero como en toda intervención quirúrgica, existe un riesgo excepcional de morir derivado del acto quirúrgico o de la situación vital de cada paciente.

En mi caso particular, el (la) doctor (a) me ha explicado que presento los siguientes riesgos adicionales:

Entiendo que si tengo necesidad de cirugía se requerirá de anestesia, cuya realización está a cargo del servicio de anestesia y sus riesgos serán valorados y considerados por el médico (a) anestesiólogo (a). Se me informa la necesidad de la presencia de un médico capacitado para la atención de mi recién nacido, quien ejercerá un acto médico independiente al cirujano obstetra.

Manifiesto que estoy satisfecha con la información recibida del médico, quien me ha dado la oportunidad de preguntar y resolver las dudas y todas ellas han sido resueltas a satisfacción. Manifiesto que he entendido sobre las condiciones y objetivos de la atención que se me va a practicar, los cuidados que debo tener, además comprendo y acepto el alcance y los riesgos justificados de posible previsión que conlleva el procedimiento médico-quirúrgico que aquí autorizo. En tales condiciones consiento que se me realice INDUCCIÓN MÉDICA DEL PARTO y acepto SE REALICE CESÁREA en caso de complicaciones o si el equipo médico lo considera necesario.

Firma del paciente

C.C. expedida en expedida en

Firma en calidad de

C.C.

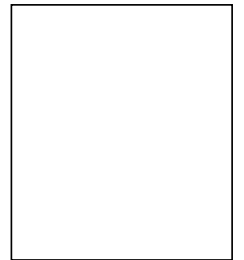

Huella índice derecho

Ciudad y fecha

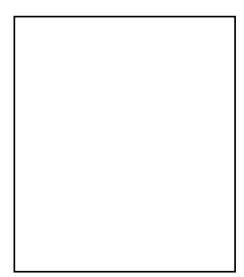

Huella índice derecho 


\section{CONSENTIMIENTO INFORMADO PARA INDUCCIÓN DE OVULACIÓN}

(En cumplimiento de la Ley 23 de 1981)

(Nombre de la paciente)

En forma voluntaria consiento en que el (la) doctor (a)

como medico tratante y los asistentes que él (ella) designe, me realicen INDUCCIÓN DE OVULACIÓN con el propósito de conseguir quedar embarazada.

Entiendo que este tratamiento consiste en la administración de diferentes medicamentos bajo supervisión médica ( ecografica y/o controles sanguíneos hormonales) con el fin de lograr que mis ovarios ovulen y se pueda producir la fecundación, para iniciar un nuevo embarazo. Entiendo además que posiblemente serán necesarios varios meses de tratamiento con cambios y ajustes de los medicamentos.

Entiendo que este tratamiento no garantiza el lograr un embarazo, pues la práctica de la medicina y la cirugía no son ciencias exactas y que por lo tanto no se puede garantizar resultados que no dependen exclusivamente del médico, debiendo el personal asignando colocar todo su conocimiento y pericia para obtener el mejor resultado.

También he entendido que existen otros tipos de tratamiento como

los cuales no acepto y voluntariamente he elegido este método médico.

Entiendo que como en todos los tratamiento médicos pueden aparecer complicaciones tales como: embarazo múltiple, hiperestimulación ovárica moderada, torsión de los ovarios o roturas de quistes foliculares, los cuales necesitarán resecciones parciales y muy raramente extracción total del ovario. La posibilidad de complicaciones severas como hiperestimulación ovárica severa con descompensación generalizada y muerte son raras, pero como en toda situación vital, existe un riesgo excepcional de morir derivado del tratamiento médico aquí indicado o de la situación vital de cada paciente.

Entiendo también que los hijos concebidos por este método, como lo normal de la población humana, tienen la posibilidad de nacer con defectos físico o mentales y que la ocurrencia de estos defectos es independiente del control médico, por consiguiente asumo la responsabilidad por las características físicas y mentales de los hijos que nazcan como resultado de este tratamiento. Entiendo también que mi embarazo tendrá los riesgos de la población general en la cual aproximadamente el $20 \%$ termina en aborto y otras complicaciones obstétricas como: parto prematuro, toxemia, ruptura de membranas, etc.

En mi caso particular, el (la) doctor (a) me ha explicado que presento los siguientes riesgos adicionales:

Manifiesto que estoy satisfecha con la información recibida del médico tratante, quien me ha dado la oportunidad de preguntar y resolver las dudas y todas ellas han sido resueltas a satisfacción. Manifiesto que he entendido sobre las condiciones y objetivos del tratamiento que se me va a practicar, además comprendo y acepto el alcance y los riesgos justificados de posible previsión que conlleva este procedimiento médico que aquí autorizo. En tales condiciones consiento que se me realice INDUCCIÓN DE OVULACIÓN.

Firma del paciente

C.C. expedida en

Firma

En calidad de

C.C. expedida en

Ciudad y fecha

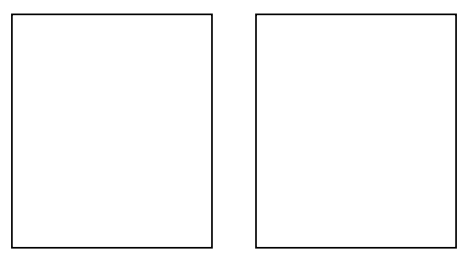

Huellas índices derechos 


\title{
CONSENTIMIENTO INFORMADO PARA INSEMINACIÓN ARTIFICIAL
}

\author{
(En cumplimiento de la Ley 23 de 1981)
}

(Nombre de la paciente)

consiento en que el (la) doctor (a)

y los asistentes que él (ella) designe, me realicen una o más INSEMINACIONES ARTIFICIALES con el propósito de conseguir quedar embarazada.

Entiendo que este tratamiento consiste en la administración de diferentes medicamentos bajo supervisión médica (ecográfica y/ o controles sanguíneos hormonales) con el fin de lograr que mis ovarios ovulen y en el momento de detectar la ovulación la colocación dentro de mi matriz del semen preparado de

para producir mi fecundación y tratar de iniciar un nuevo embarazo. Posiblemente serán necesarios varios meses de tratamiento y cambios y ajustes de los medicamentos.

Entiendo que este tratamiento no garantiza el lograr un embarazo, pues la práctica de la medicina y cirugía no son una ciencia exacta y por lo tanto los que la siguen no pueden garantizar resultados que no dependen exclusivamente del medico, debiendo ellos colocar toda su pericia en tratar de obtener los mejores resultados.

También he entendido que existen otros tipos de tratamiento como

los cuales no acepto y voluntariamente he elegido este método.

Entiendo que pueden aparecer complicaciones tales como: mareo, dolor, infecciones, embarazo múltiple, hiperestimulación ovárica moderada, torsión de los ovarios o roturas de quistes foliculares, los cuales necesitarán resecciones parciales y muy raramente extracción total del ovario, y embarazo ectópico que necesitará la resección parcial de la trompa. La posibilidad de complicaciones como contaminación con PAPILOMA VIRUS o SIDA (cuando el semen es de donante), hiperestimulación ovárica severa con descompensación generalizada y muerte son raras, pero como en toda situación vital, existe un riesgo excepcional de morir derivado del tratamiento médico aquí indicado o de la situación vital de cada paciente.

Entiendo también que los hijos concebidos por este método, como lo normal de la población humana, tienen posibilidad de nacer con defectos físico o mentales y que la ocurrencia de estos defectos es independiente del control médico, por consiguiente asumo la responsabilidad por las características físicas y mentales de los hijos que nazcan como resultado de este tratamiento. Entiendo también que mi embarazo tendrá los riesgos de la población general en la cual aproximadamente el 20\% termina en aborto y otras complicaciones obstétricas como: parto prematuro, toxemia, ruptura de membranas, etc.

En mi caso particular, el (la) doctor (a) me ha explicado que presento los siguientes riesgos adicionales:

Manifiesto que estoy satisfecha con la información recibida del médico tratante, quien me ha dado la oportunidad de preguntar y resolver las dudas a satisfacción. Manifiesto que he entendido sobre las condiciones y objetivos del tratamiento que se me va a practicar, además comprendo y acepto el alcance y los riesgos justificados de posible previsión que conlleva el procedimiento médico que aquí autorizo. En tales condiciones consiento que se me realice INSEMINACIÓN ARTIFICIAL CON EL SEMEN DE

Firma del paciente

C.C.

(Nombre)

En calidad de consiento y acepto el acto médico de inseminación artificial arriba descrito, acepto sus riesgos y complicaciones, y estoy de acuerdo en que los niños así concebidos o nacidos, serán mis legítimos hijos y herederos.

Firma

C.C.

Ciudad y fecha 


\section{CONSENTIMIENTO INFORMADO PARA LAPAROTOMÍA EXPLORADORA}

(En cumplimiento de la Ley 23 de 1981)

(Nombre de la paciente)

consiento en que el (la) doctor (a)

como cirujano(a) y el ayudante que él (ella) designe, me realicen cirugía LAPAROTOMÍA EXPLORADORA ante la dificultad para llegar a un diagnóstico correcto a través de otras pruebas diagnosticas por presentar:

Entiendo que esta cirugía consiste básicamente en la apertura de la cavidad abdominal por medio de una herida en la cara anterior del abdomen y revisión de los órganos abdominales y pélvicos. Según los hallazgos se hará extirpación de las estructuras que estén indicadas (apéndice, epiplón, anexos, ovarios, matriz, etc.) drenaje de sangre o pus. En caso de encontrar un tumor de sospecha cancerosa se podrá tomar biopsia por congelación para decidir según su resultado el tipo de intervención que debe hacerse como extirpación de todo el aparato genital con cadenas ganglionares o simplemente cirugía reductora (sacar parte del tumor). En caso de encontrase muchos órganos lesionados será necesario la participación de otros especialistas (cirujano, urólogo, etc.)

Esta cirugía no garantiza totalmente la identificación y/o desaparición de mi problema, ni evita que en el futuro sean necesarias nuevas intervenciones para solucionar problemas residuales o complicaciones. Se me ha explicado que la garantía no es total pues la práctica de la medicina y cirugía no son una ciencia exacta y que por lo tanto los que la siguen no pueden garantizar resultados que no dependen exclusivamente del médico, debiendo ellos colocar todos sus conocimientos y pericia en obtener los mejores resultados.

También he entendido que existen otros métodos diagnósticos como

los cuales no acepto y voluntariamente he elegido este método quirúrgico.

Entiendo que como en toda intervención quirúrgica pueden aparecer complicaciones tales como: náuseas, vómito, dolor, inflamación, moretones, seromas (acumulación de líquido en la cicatriz), granulomas (reacción a cuerpo extraño o sutura), queloide (crecimiento excesivo de la cicatriz), hematomas (acumulación de sangre), sangrado o hemorragias con la posible necesidad de transfusión (intra o posoperatoria), infecciones con posible evolución febril (urinarias, de piel, abscesos, peritonitis) reacciones alérgicas, anemia, perforaciones involuntarias de órganos vecinos como vejiga, intestino, colon, vasos sanguíneos, fístulas urinarias o intestinales, hernias abdominales, adherencias, obstrucción intestinal por bridas. En caso de ser necesaria la extracción de la matriz se quedará con una permanente imposibilidad para tener hijos y si se extraen los ovarios se entrará en menopausia requiriendo reemplazo hormonal de acuerdo a criterio médico. La posibilidad de complicaciones severas como septisemia (infección generalizada), colostomía, ligadura de grandes vasos o de uréter, trombosis o muerte son raras, pero como en toda intervención quirúrgica, existe un riesgo excepcional de morir derivado del acto quirúrgico o de la situación vital de cada paciente.

En mi caso particular, el (la) doctor (a) me ha explicado que presento los siguientes riesgos adicionales:

Entiendo que para esta cirugía se necesita anestesia, cuyo riesgo será valorado, se realizara y será responsabilidad del servicio de anestesia.

Entiendo que la pieza o piezas extirpadas se someterán a estudio anatomopatológico inmediatamente por congelación o posteriormente según criterio médico resultado final e informarlo al médico. en teniendo que estar pendiente de su

Manifiesto que estoy satisfecha con la información recibida del médico tratante, quien lo ha hecho en un lenguaje claro y sencillo, y me ha dado la oportunidad de preguntar y resolver las dudas a satisfacción. Manifiesto que yo he entendido sobre las condiciones y objetivos de la cirugía que se me va a practicar, los cuidados que debo tener antes y después de ella, además comprendo y acepto el alcance y los riesgos justificados de posible previsión que conlleva este procedimiento quirúrgico que aquí autorizo. En tales condiciones consiento que se me realice LAPAROTOMÍA EXPLORADORA.

Firma del paciente

C.C.

Ciudad y fecha

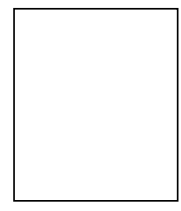

Huella índice derecho 


\section{CONSENTIMIENTO INFORMADO PARA LEGRADO}

(En cumplimiento de la Ley 23 de 1981)

(Nombre)

en forma voluntaria consiento en que el (la) doctor (a) me realice LEGRADO UTERINO por presentar

Entiendo que ese procedimiento consiste básicamente en limpiar la cavidad endometrial (parte interna del útero o matriz) por vía vaginal a través del cuello uterino. A veces es necesario un segundo legrado para completar esta evacuación sobre todo en los casos de aborto retenido. La intervención consiste en dilatar el cuello uterino (algunas veces no hay necesidad de dilatar por encontrarse el cuello ya entreabierto) y la extracción de restos ovulares o endometriales con pinzas, legras romas y cortantes o bien por aspiración. En algunos casos es necesario colocar el día anterior a la evacuación tallos de laminaria para lograr dilatación cervical progresiva o la aplicación de oxitocina o sustancias prostaglandínicas.

Se me ha explicado que la garantía no es total pues la práctica de la medicina y cirugía no son una ciencia exacta, debiendo mi médico colocar todo su conocimiento y su pericia en buscar obtener el mejor resultado.

Entiendo que como en toda intervención quirúrgica y por causas independiente del actuar de mi médico se pueden presentar complicaciones que podrían requerir tratamientos complementarios tanto médicos como quirúrgicos, tales como: infecciones (urinarias, uterinas, de las trompas...), hemorragias con la posible necesidad de transfusión sanguínea intra o posoperatoria, perforación de útero, persistencia de restos, abscesos, dolor pélvico, adherencias (pélvicas o intrauterinas). También se me informa la posibilidad de complicaciones severas como infertilidad, infecundidad, pelvi peritonitis, trombosis que, aunque son poco frecuentes, representan como en toda intervención quirúrgica un riesgo excepcional de perder la vida, derivado del acto quirúrgico o de la situación vital de cada paciente.

En mi caso particular, el (la) doctor (a) me ha explicado que presento los siguientes riesgos adicionales:

Entiendo que para esta cirugía se necesita anestesia, la cual se evaluará y realizará por el servicio de anestesia.

Entiendo que el material que se saque se someterá a estudio anatomopatológico posterior en siendo mi deber el reclamar su resultado e informarlo al médico.

He entendido los cuidados que debo tener antes y después, estoy satisfecha con la información recibida del médico tratante, quien me ha dado la oportunidad de preguntar y resolver las dudas a satisfacción, además comprendo y acepto el alcance y los riesgos justificados de posible previsión que conlleva el procedimiento quirúrgico que aquí autorizo. En tales condiciones consiento que se me realice LEGRADO UTERINO.

Firma del paciente

C.C.

Ciudad y fecha

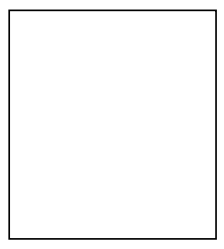

Huella índice derecho 


\title{
CONSENTIMIENTO INFORMADO PARA CIRUGIA COMO TRATAMIENTO DE INFERTILIDAD
}

\author{
(En cumplimiento de la Ley 23 de 1981)
}

(Nombre)

en forma voluntaria consiento en que el (la) doctor (a)

como cirujano(a) y el ayudante que el (ella) designe me realicen CIRUGIA COMO TRATAMIENTO PARA INFERTILIDAD por presentar

Entiendo que esta cirugía consiste básicamente en la adecuación de los órganos pélvicos para concebir un embarazo, la cual puede realizarse por: laparoscopia, histeroscopia, microlaparotomía(pequeña herida), o comúnmente vía abdominal (herida en la cara anterior del abdomen), utilizando a veces instrumentos ópticos de aumento (lupas, microscopios, etc.). Este procedimiento depende de mi problema en particular pero frecuentemente consiste en liberar adherencia o abrir la terminación de la trompa, para recuperar la permeabilidad de las trompas de Falopio. A veces es necesario la nueva unión de las trompas con una sutura muy fina bajo visión microscópica (reanastomosis).

Esta cirugía no garantiza el lograr un embarazo, ni evita la posibilidad de que aparezcan nuevas adherencias o se obstruyan nuevamente las trompas en el futuro. Se me ha explicado y entiendo que la garantía no es total pues la práctica de la medicina y la cirugía no son ciencias exactas, por lo que mi médico debe todo su conocimiento y su pericia en buscar los mejores resultados con el objetivo de mejorar las posibilidades que tengo de lograr un embarazo.

También he entendido que existen otros tipos de tratamiento como

los cuales no acepto y voluntariamente he elegido este método quirúrgico.

Entiendo que como en toda intervención quirúrgica y por causas independientes del actuar de mi médico se pueden presentar complicaciones comunes y potencialmente serias que podrían requerir tratamientos complementarios, tanto médicos como quirúrgicos, tales como: náuseas, vómito, dolor, inflamación, moretones, seromas (acumulación de líquido en la cicatriz), granulomas (reacción a cuerpo extraño o sutura), queloide (crecimiento excesivo de la cicatriz), hematomas (acumulación de sangre), apraxia (cambios en la sensibilidad de la piel), cistitis, retención urinaria, sangrado o hemorragias con la posible necesidad de transfusión (intra o posoperatoria), infecciones con posible evolución febril (urinarias, de pared abdominal, pélvicas...), reacciones alérgicas, irritación frénica, anemia, heridas involuntarias en vasos sanguíneos, vejiga, intestino u otros órganos, dolor pélvico, reaparición de adherencias, hidrosalpinx, reobstrucción de las trompas, fístulas, eventración (hernias en la cicatriz) o ruptura de embarazo ectópico. También se me informa la posibilidad de complicaciones severas como pelvi peritonitis, choque hemorrágico, trombosis, que aunque son poco frecuentes representan como en toda intervención quirúrgica un riesgo excepcional de perder la vida derivado del acto quirúrgico o de la situación vital de cada paciente.

En mi caso particular, el (la) doctor (a) me ha explicado que presento los siguientes riesgos adicionales:

Entiendo que para esta cirugía se necesita anestesia, la cual se evaluará y realizará por el servicio de anestesia.

Entiendo que si es necesario extraer algún tejido, se someterá a estudio anatomopatológico posterior en siendo mi deber el reclamar el resultado e informarlo al médico.

He entendido sobre las condiciones y objetivos de la cirugía que se me va a practicar, los cuidados que debo tener antes y después de ella, estoy satisfecha con la información recibida del médico tratante quien lo ha hecho en un lenguaje claro y sencillo, y me ha dado la oportunidad de preguntar y resolver las dudas a satisfacción, además comprendo y acepto el alcance y los riesgos justificados de posible previsión que conlleva este procedimiento quirúrgico que aquí autorizo. En tales condiciones consiento que se me realice CIRUGÍA PARA INFERTILIDAD.

Firma del paciente

C.C. expedida en

Ciudad y fecha

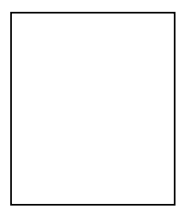

Huella índice derecho 


\title{
CONSENTIMIENTO INFORMADO PARA MIOMECTOMÍA
}

\author{
(En cumplimiento de la Ley 23 de 1981)
}

(Nombre)

en forma voluntaria consiento en que el (la) doctor (a)

como cirujano(a) y el ayudante que el (ella) designe, me realicen cirugía DE MIOMECTOMÍA como tratamiento para miomatosis uterina.

Entiendo que esta cirugía consiste básicamente en la extirpación de uno o más miomas tratando de conservar el útero o matriz, la cual puede realizarse por: laparoscopia, histeroscopia, microlaparotomía(pequeña herida), o comúnmente vía abdominal (herida en la cara anterior del abdomen). Entiendo que existe la posibilidad según el estado de los miomas de que el cirujano se vea en la necesidad de proceder a una HISTERECTOMÍA, cirugía que consiste en la extirpación del útero con o sin cuello, lo que supone la imposibilidad de tener hijos, así como la ausencia de menstruaciones. La histerectomía puede llevar asociadas la extirpación de los anexos (ovarios y trompas) según edad, patología asociada y criterio médico en el momento de la intervención. Al extirpar los ovarios se instaura la menopausia, pudiendo recibir terapia hormonal sustitutiva posteriormente, según indicación médica.

Esta cirugía no garantiza la desaparición total de los miomas, ni evita la posibilidad de que aparezcan nuevos miomas en el futuro. Se me ha explicado y entiendo que la garantía no es total pues la práctica de la medicina y cirugía no son una ciencia exacta, debiendo mi médico colocar todo su conocimiento y su pericia en buscar obtener el mejor resultado.

También he entendido que existen otros tipos de tratamiento como hormonales para las hemorragias y para disminuir temporalmente su tamaño, y analgésicos para el dolor, los cuales no acepto y voluntariamente he elegido este método quirúrgico de MIOMECTOMÍA.

Entiendo que como en toda intervención quirúrgica y por causas independientes del actuar de mi médico se pueden presentar complicaciones comunes y potencialmente serias que podrían requerir tratamientos complementarios tanto médicos como quirúrgicos, siendo las complicaciones de la miomectomia: nauseas, vomito, dolor, inflamación, moretones, seromas (acumulación de liquido en la cicatriz), granulomas (reacción a cuerpo extraño o sutura), queloide (crecimiento excesivo de la cicatriz), hematomas(acumulación de sangre), cistitis, retención urinaria, sangrado o hemorragias con la posible necesidad de transfusión (intra o postoperatoria), infecciones (urinarias, de pared abdominal, pélvicas...), reacciones alérgicas, irritación frénica, anemia, cambios en la sensibilidad de la piel, heridas o quemadura por bisturí eléctrico involuntarias en vasos sanguíneos, vejiga u otros órganos, dolor pélvico, adherencias, fístulas, eventración (hernias en la cicatriz), prolapsos (descensos) de cúpula vaginal, cuando se saca la matriz, problemas en los ovarios cuando se conservan los anexos o ruptura uterina en un embarazo posterior. También se me informa la posibilidad de complicaciones severas como pelvi peritonitis, trombosis o hemorragia severa con choque, que aunque son poco frecuentes, representan como en toda intervención quirúrgica un riesgo excepcional de perder la vida, derivado del acto quirúrgico o de la situación vital de cada paciente. Entiendo claramente que si es necesario realizar HISTERECTOMÍA esta operación me dejará con una permanente incapacidad para tener hijos y acepto la infertilidad producida por ella.

En mi caso particular, el (la) doctor (a) me ha explicado que presento los siguientes riesgos adicionales:

Entiendo que para esta cirugía, se necesita anestesia, la cual se evaluará y realizará por el servicio de anestesia.

Entiendo que la pieza extirpada se someterá a estudio anatomopatologico posterior en siendo mi deber el reclamar su resultado e informarlo al médico.

He entendido las condiciones y objetivos de la cirugía que se me va a practicar, los cuidados que debo tener antes y después de ella, estoy satisfecha con la información recibida del médico tratante quien lo ha hecho en un lenguaje claro y sencillo, y me ha dado la oportunidad de preguntar y resolver las dudas a satisfacción, además comprendo y acepto el alcance y los riesgos justificados de posible previsión que conlleva el procedimiento quirúrgico que aquí autorizo. En tales condiciones consiento que se me realice MIOMECTOMÍA.

Firma del paciente

C.C. expedida en

Ciudad y fecha

Huella índice derecho 


\section{CONSENTIMIENTO INFORMADO PARA CIRUGÍA DE OCLUSIÓN TUBÁRICA}

(En cumplimiento de la Ley 23 de 1981)

(Nombre )

en forma voluntaria consiento en que el (la) doctor (a)

como cirujano(a) y el ayudante que él (ella) designe, me realicen cirugía DE OCLUSIÓN TUBÁRICA con el objeto de impedir un nuevo embarazo.

Entiendo que esta cirugía consiste básicamente en la interrupción de la continuidad de las trompas de Falopio, la cual puede realizarse por laparoscopia, microlaparotomía (pequeña apertura en la pared abdominal anterior), vaginal o durante una cesárea. Existe una muy pequeña posibilidad de embarazarme después de que la cirugía haya sido realizada. Se me ha explicado y entiendo que la garantía no es total pues la práctica de la medicina y la cirugía no son ciencias exactas, debiendo mi médico colocar todo su conocimiento y su pericia en buscar obtener el mejor resultado.

También entiendo que existen otros tipos de contracepción (métodos de evitar embarazo) que son temporales y reversibles tales como: métodos hormonales (píldoras anticonceptivas, inyecciones, norplant), dispostivos intrauterinos, métodos de barrera y naturales, los cuales no acepto y voluntariamente he elegido este método quirúrgico irreversible. Entiendo claramente que esta operación me dejará con una permanente incapacidad para tener hijos y acepto la infertilidad producida por ella.

Entiendo que como en toda intervención quirúrgica y por causas independientes del actuar de mi médico se pueden presentar complicaciones como: náuseas, vómito, dolor, inflamación, moretones, seromas (acumulación de líquido en la cicatriz), granulomas (reacción a cuerpo extraño o sutura), queloide (crecimiento excesivo de la cicatriz), hematomas (acumulación de sangre), cistitis, retención urinaria, sangrado o hemorragias con la posible necesidad de transfusión (intra o posoperatoria), infecciones (urinarias, de pared abdominal, pélvicas...), reacciones alérgicas, irritación frénica, anemia, cambios en la sensibilidad de la piel, heridas o quemadura por bisturí eléctrico involuntarias en vasos sanguíneos, vejiga u otros órganos, dolor pélvico, adherencias, eventración (hernias en la cicatriz), hidrosalpinx (acumulación de líquido en las trompas seccionadas) o embarazo ectópico. También se me informa la posibilidad de complicaciones severas como pelvi peritonitis, trombosis o hemorragia severa con choque que, aunque son poco frecuentes, representan como en toda intervención quirúrgica un riesgo excepcional de perder la vida, derivado del acto quirúrgico o de la situación vital de cada paciente.

En mi caso particular el (la) doctor(a) me ha explicado que presento los siguientes riesgos adicionales:

Entiendo que para esta cirugía se necesita anestesia, la cual se evaluará y realizará por el servicio de anestesia.

He entendido las condiciones y los objetivos de la cirugía que se me va a practicar, los cuidados que debo tener antes y después de ella, estoy satisfecha con la información recibida del médico tratante quien lo ha hecho en un lenguaje claro y sencillo, y me ha dado la oportunidad de preguntar y resolver las dudas a satisfacción, además comprendo y acepto el alcance y los riesgos justificados de posible previsión que conlleva el procedimiento quirúrgico que aquí autorizo. En tales condiciones consiento que se me realice CIRUGÍA DE OCLUSIÓN TUBÁRICA.

Firma del paciente

C.C. expedida en

Firma

En calidad de

C.C. expedida en

Ciudad y fecha

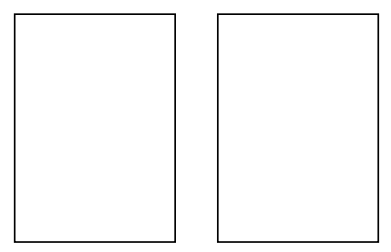

Huellas índices derechos 


\section{CONSENTIMIENTO INFORMADO PARA RESECCIÓN DE MASA EN SENO}

(En cumplimiento de la Ley 23 de 1981)

(Nombre)

en forma voluntaria consiento en que el (la) doctor (a)

como cirujano(a) y el (la)ayudante que él (ella) designe, me realicen cirugía DE RESECCION DE MASA EN SENO O BIOPSIA DE SENO ante la dificultad para llegar a un diagnóstico a través de otras pruebas diagnósticas, por presentar una masa en el seno y/o hallazgos en la mamografía.

Entiendo que esta cirugía consiste básicamente en sacar por medio de una apertura de la piel de mi seno las posibles estructuras comprometidas en el problema y un examen de los tejidos por un patólogo para ayudar a llegar al diagnóstico de mi enfermedad.

Se me ha explicado y entiendo que como en todo acto médico no puede existir garantía en la identificación y desaparición de mi problema, ni este procedimiento evita la posibilidad de que aparezcan nuevas masas en el futuro, pues la práctica de la medicina y cirugía no son una ciencia exacta, debiendo mi médico colocar todo su conocimiento y su pericia en obtener el mejor resultado.

También he entendido que existen otros métodos diagnósticos como biopsia por aspiración, mamotron o los cuales no acepto y voluntariamente he elegido este método qui-

rúrgico.

Entiendo que para esta cirugía se necesita anestesia, la cual se evaluará y realizará por el servicio de anestesia.

Entiendo que la pieza extirpada se someterá a estudio anatomopatológico posterior en siendo mi deber el reclamar su resultado e informarlo al médico.

Entiendo que como en toda intervención quirúrgica y por causas independientes del actuar de mi médico se pueden presentar complicaciones comunes y potencialmente serias que podrían requerir tratamientos complementarios, tanto médicos como quirúrgicos, tales como: dolor, inflamación, moretones, seromas (acumulación de líquido en la cicatriz), necrosis de la piel, granulomas (reacción a cuerpo extraño o sutura), queloide (crecimiento excesivo de la cicatriz), hematomas (acumulación de sangre), sangrado o hemorragias con la posible necesidad de transfusión (intra o posoperatoria), infecciones con posible evolución febril y abscesos, reacciones alérgicas, dificultad con una futura lactancia, deformación del seno. También se me informa la posibilidad de complicaciones severas como septisemia (infección generalizada), o trombosis que, aunque son poco frecuentes, representan como en toda intervención quirúrgica un riesgo excepcional de perder la vida derivado del acto quirúrgico o de la situación vital de cada paciente.

En mi caso particular, el (la) doctor (a) me ha explicado que presento los siguientes riesgos adicionales:

He entendido sobre las condiciones y objetivos de la cirugía que se me va a practicar, los cuidados que debo tener antes y después de ella, estoy satisfecha con la información recibida del medico tratante, quien lo ha hecho en un lenguaje claro y sencillo y me ha dado la oportunidad de preguntar y resolver las dudas y todas ellas han sido resueltas a satisfacción, además comprendo y acepto el alcance y los riesgos justificados de posible previsión que conlleva este procedimiento quirúrgico que aquí autorizo. En tales condiciones consiento que se me realice RESECCIÓN DE MASA EN SENO O BIOPSIA DE SENO.

Firma del paciente

C.C. expedida en

Ciudad y fecha

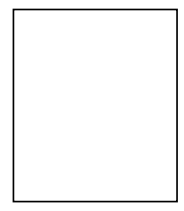

Huella índice derecho 


\section{CONSENTIMIENTO INFORMADO PARA CIRUGIA DE RELAJACIÓN DE PISO PÉLVICO}

(En cumplimiento de la Ley 23 de 1981)

(Nombre)

en forma voluntaria, consiento en que el (la) doctor (a)

como cirujano(a) y el ayudante que el (ella) designe, me realicen CIRUGÍA PARA RELAJACIÓN DEL PISO PÉLVICO como tratamiento para prolapso vaginal.

Entiendo que esta cirugía consiste básicamente en la reconstrucción del piso pélvico que incluye tratamiento del descenso de vejiga y recto y/o lesión preexistente en vulva, vagina y periné. Esta cirugía se puede realizar toda vía vaginal, vía abdominal o en forma combinada abdominal y vaginal.

Se me ha explicado y entiendo que como en todo acto médico no puede existir garantía en los resultados, pues la práctica de la medicina y cirugía no son una ciencia exacta, debiendo mi médico colocar todo su conocimiento y su pericia en obtener el mejor resultado.

También he entendido que existen otros tipos de tratamientos como: pesarios, ejercicio y medicamentos para aliviar los síntomas los cuales no acepto y voluntariamente he elegido este método quirúrgico.

Entiendo que como en toda intervención quirúrgica y por causas independientes del actuar de mi médico se pueden presentar complicaciones comunes y potencialmente serias que podrían requerir tratamientos complementarios, tanto médicos como quirúrgicos, tales como: náuseas, vómito, dolor, inflamación, granulomas en vagina (reacción a cuerpo extraño o sutura), hematomas, cistitis, retención urinaria (imposibilidad para orinar), sangrado o hemorragias con la posible necesidad de transfusión (intra o posoperatoria), infecciones con posible evolución febril (abscesos, urinarias, de pared abdominal), reacciones alérgicas, anemia, heridas o lesiones involuntarias en vejiga, recto u otros órganos, fístulas (escape involuntario de orina o materia fecal por la vagina), defectos de cicatrización que pueden producir estrechamientos vulvovaginales, dispareunia (dolor con las relaciones sexuales), persistencia de la incontinencia urinaria y de los prolapsos (descensos). También se me informa la posibilidad de complicaciones severas como pelvi peritonitis, septicemia (infección generalizada), trombosis o muerte, que aunque son poco frecuentes representan como en toda intervención quirúrgica un riesgo excepcional de perder la vida, derivado del acto quirúrgico o de la situación vital de cada paciente.

En mi caso particular el (la) doctor(a) me ha explicado que presento los siguientes riesgos adicionales:

Entiendo que para esta cirugía se necesita anestesia, la cual se evaluará y realizará por el servicio de anestesia.

He entendido las condiciones y objetivos de la cirugía que se me va a practicar, los cuidados que debo tener antes y después de ella, estoy satisfecha con la información recibida del médico tratante, quien lo ha hecho en un lenguaje claro y sencillo, y me ha dado la oportunidad de preguntar y resolver las dudas a satisfacción, además comprendo y acepto el alcance y los riesgos justificados de posible previsión que conlleva el procedimiento quirúrgico que aquí autorizo. En tales condiciones consiento que se me realice CIRUGÍA PARA RELAJACIÓN DE PISO PÉLVICO.

Firma del paciente

C.C. expedida en

Ciudad y fecha

Huella índice derecho 


\section{DISENTIMIENTO INFORMADO PARA REMISIÓN}

(En cumplimiento de la Ley 23 de 1981)

Institución

Historia clínica

(Nombre)

RECHAZO SER REMITIDA A:

El doctor(a)

me ha explicado claramente y yo he entendido que la remisión es necesaria por razones médicas.

Igualmente el doctor(a) me ha explicado que los riesgos de rechazar la remisión y de mi decisión de permanecer en esta institución son:

ACEPTO ESTOS RIESGOS BAJO MI PROPIA RESPONSABILIDAD.

Firma del paciente

c.c. expedida en

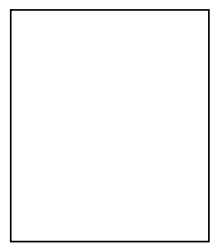

Firma

c.c. expedida en

En calidad de:

Firma del médico

Reg. No.

Ciudad, fecha y hora
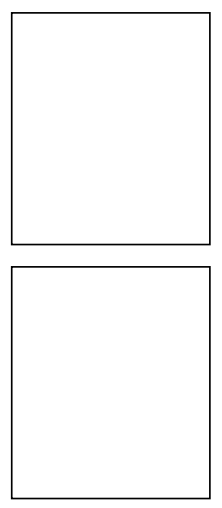

Huellas índices derechos 


\section{CONSENTIMIENTO INFORMADO PARA CIRUGÍA}

(En cumplimiento de la Ley 23 de 1981)

(Nombre)

en forma voluntaria consiento en que el (la) doctor (a)

como cirujano(a) y el ayudante que el (ella) designe me realicen

COMO TRATAMIENTO PARA

Entiendo que esta cirugía consiste básicamente en

Esta cirugía no garantiza totalmente los resultados esperados. Se me ha explicado y entiendo que la garantía no es total pues la práctica de la medicina y cirugía no son una ciencia exacta, debiendo mi médico colocar todo su conocimiento y su pericia en buscar los mejores resultados con el objetivo de mejorar el problema por el cual consulté.

También he entendido que existen otros tipos de tratamiento como

los cuales no acepto y voluntariamente he elegido este método quirúrgico.

Entiendo que como en toda intervención quirúrgica y por causas independientes del actuar de mi médico se pueden presentar complicaciones comunes y potencialmente serias que podrían requerir tratamientos complementarios, tanto médicos como quirúrgicos, siendo las complicaciones más frecuentes: náuseas, vómito, dolor, inflamación, moretones, seromas (acumulación de líquido en la cicatriz), granulomas (reacción a cuerpo extraño o sutura), queloide (crecimiento excesivo de la cicatriz), hematomas(acumulación de sangre), apraxias (cambios en la sensibilidad de la piel), cistitis, retención urinaria, sangrado o hemorragias con la posible necesidad de transfusión (intra o posoperatoria), infecciones con posible evolución febril (urinarias, de pared abdominal, pélvicas...), reacciones alérgicas, irritación frénica, anemia, heridas involuntarias en vasos sanguíneos, vejiga, intestino u otros órganos, eventración (hernias en la cicatriz. Existen otros riesgos como:

También se me informa la posibilidad de complicaciones severas como pelviperitonitis, choque hemorrágico o trombosis que, aunque son poco frecuentes, representan como en toda intervención quirúrgica un riesgo excepcional de perder la vida derivado del acto quirúrgico o de la situación vital de cada paciente.

En mi caso particular, el (la) doctor (a) me ha explicado que presento los siguientes riesgos adicionales:

Entiendo que para esta cirugía se necesita anestesia, la cual se evaluará y realizará por el servicio de anestesia.

Entiendo que si es necesario extraer algún tejido, se someterá a estudio anatomopatológico posterior en siendo mi deber reclamar el resultado e informarlo al médico.

He entendido las condiciones y objetivos de la cirugía que se me va a practicar, los cuidados que debo tener antes y después de ella, estoy satisfecha con la información recibida del médico tratante quien lo ha hecho en un lenguaje claro y sencillo, y me ha dado la oportunidad de preguntar y resolver las dudas a satisfacción, además comprendo y acepto el alcance y los riesgos justificados de posible previsión que conlleva el procedimiento quirúrgico que aquí autorizo. En tales condiciones consiento que se me realice CIRUGÍA.

Firma del paciente Firma del esposo, compañero

C.C. C.C.

Ciudad y fecha 


\section{REFERENCIAS BIBLIOGRÁFICAS}

1. Código de Ética Médica. Ley 23 de 1981.

2. Código de Ética Médica, Decreto 3380 de 1981.
3. Mondragón AL. Formatos para preparación de informe de histeroscopia. Rev Colomb Obstet Ginecol 49(4).

4. Parra G, et al. Formatos de consentimiento informado. Una propuesta. SCOG. 